\title{
KINEMATICAL EVOLUTION OF THE PAIRWISE VELOCITY FIELD OF GALAXIES
}

\author{
NAOKI SETO \\ Department of Physics, Kyoto University, Kyoto 606-01 \\ AND \\ JUN'ICHI YOKOYAMA \\ Yukawa Institute for Theoretical Physics, Kyoto University, \\ Kyoto 606-01
}

It has been a long-standing cosmological issue to explain why the statistical distribution of the radial pairwise peculiar velocity of galaxies has an exponential shape rather than the Gaussian, as suggested by observations and explicitly shown by results of $\mathrm{N}$-body simulations. Previous explanations on the exponential distribution are based on highly ronlinear dynamics of galactic systems $\left(R<1 h^{-1} \mathrm{Mpc}\right)$. That is, the exponential feature appears as a result of superposition of Gaussian velocity distributions in clumps with various velocity dispersions. Hence although this model is suitable to account for a symmetric exponential distribution with vanishing mean net relative velocity, a more elaborate treatment is necessary in order to reproduce an asymmetric exponential distribution with its peak at a negative relative velocity as observed in numerical simulations.

Here we trace evolution of the pairwise velocity field in terms of the Zel'dovich approximation assuming that the primordial density fluctuations are Gaussian distributed. We find that by the effect of displacement, the nontrivial exponential distribution is realized as a transient phenomena under a generic spectrum of initial fluctuations. Our formula also predicts a skewed distribution, which is observed in $\mathrm{N}$-body simulation

\section{References}

Seto, N. and Yokoyama, J. (1998), ApJ, in press (Jan 10 issue, astro-ph/9707312)

Sheth, R. K. (1996), MNRAS, 279, 1311

Zurek, W. H. et al. (1994) $A p J, 431,559$ 